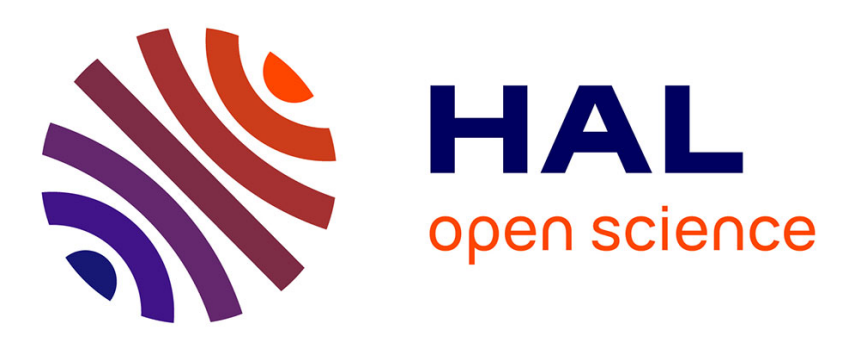

\title{
Une intervention psychosociologique dans le contexte de la Martinique: le rôle clef du dispositif
}

Nicolas Lamic

\section{To cite this version:}

Nicolas Lamic. Une intervention psychosociologique dans le contexte de la Martinique: le rôle clef du dispositif. Nouvelle revue de psychosociologie, 2007, 10.3917/nrp.003.0181 . hal-01664274

\section{HAL Id: hal-01664274 \\ https://hal.univ-antilles.fr/hal-01664274}

Submitted on 14 Dec 2017

HAL is a multi-disciplinary open access archive for the deposit and dissemination of scientific research documents, whether they are published or not. The documents may come from teaching and research institutions in France or abroad, or from public or private research centers.
L'archive ouverte pluridisciplinaire $\mathbf{H A L}$, est destinée au dépôt et à la diffusion de documents scientifiques de niveau recherche, publiés ou non, émanant des établissements d'enseignement et de recherche français ou étrangers, des laboratoires publics ou privés. 


\section{CA I R N}

chercher : repérer : avancer

Cet article est disponible en ligne à l'adresse :

http://www.cairn.info/article.php?ID REVUE=NRP\&ID NUMPUBLIE=NRP 003\&ID ARTICLE=NRP $003 \quad 0181$

Une intervention psychosociologique dans le contexte de la Martinique : le rôle clef du dispositif

par Nicolas LAMIC

\section{érès | Nouvelle revue de psychosociologie}

\section{7/1 -}

ISSN 1951-9532 | ISBN 2-7492-0726-1| pages 181 à 194

\section{Pour citer cet article :}

- Lamic N., Une intervention psychosociologique dans le contexte de la Martinique : le rôle clef du dispositif, Nouvelle revue de psychosociologie 2007/ 1, , p. 181-194.

Distribution électronique Cairn pour érès.

(C) érès. Tous droits réservés pour tous pays.

La reproduction ou représentation de cet article, notamment par photocopie, n'est autorisée que dans les limites des conditions générales d'utilisation du site ou, le cas échéant, des conditions générales de la licence souscrite par votre établissement. Toute autre reproduction ou représentation, en tout ou partie, sous quelque forme et de quelque manière que ce soit, est interdite sauf accord préalable et écrit de l'éditeur, en dehors des cas prévus par la législation en vigueur en France. Il est précisé que son stockage dans une base de données est également interdit. 


\section{études}

\section{Une intervention psychosociologique dans le contexte de la Martinique : le rôle clef du dispositif}

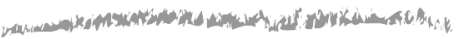

\section{Nicolas LAMIC}

Les deux expériences brièvement relatées dans cet article montrent comment toute action de changement dans des entreprises martiniquaises et guadeloupéennes se heurte aux résistances provoquées par les structures et les clivages de nature raciale profondément ancrés dans la culture antillaise, hérités du colonialisme et de l'esclavage, qui conditionnent dans l'entreprise les identités, les rapports hiérarchiques, les modes de division du travail, et les conduites individuelles et collectives.

À moins de se limiter à un rôle de prestation de services maintenant le statu quo, et pour être en mesure de traiter les racines des conflits à l'origine de la demande d'intervention, celle-ci doit être conduite de façon à surmonter ces résistances, d'autant plus difficiles à cerner et à traiter que les clivages raciaux sont niés, qu'ils constituent un tabou. Elles condi- tionnent l'acceptation ou le refus des dispositifs proposés par le consultant, la façon dont ils sont mis en place, tout en affectant également le consultant ou l'équipe de consultants, la place et le statut qui leur est reconnu, les relations à leur égard, la façon dont ils sont considérés.

Dans cette situation difficile, le consultant est mis en question dans son identité personnelle et professionnelle. Afin de pouvoir maintenir néanmoins la position de tiers requise par la démarche $\mathrm{d}^{\prime}$ intervention psychosociologique, il doit $\mathrm{s}^{\prime}$ appuyer sur des valeurs et des convictions d'ordre éthique, mais aussi sur de solides bases théoriques (théorie de l'intervention, analyses de la société martiniquaise et de son histoire).

Soumis aux fortes pressions s'exerçant sur lui, il doit également être extrêmement vigilant quant à son mode d'implication personnelle dans la situa-

Nicolas Lamic, psychosociologue, enseignant à l'université des Antilles et de la Guyane, directeur du cabinet Horizons conseils.

nicolas.lamic@horizonsconseils.com 
tion, au risque de s'identifier à l'une ou l'autre partie, à la nécessité de conserver la distance nécessaire.

Cela est d'autant plus difficile lorsque le consultant appartient lui-même à la communauté antillaise noire, étant partagé entre son identification à cette communauté d'appartenance et son identité en tant que citoyen français, formé en France et identifié à la culture française.

C'est ainsi que dans nos interventions, nous avons souvent été confrontés à la question du retentissement des difficultés liées aux stéréotypes raciaux que rencontrent ces collectifs organisationnels quant à notre positionnement comme consultant. Dans ce travail, I'insistance sur la place que tient la recherche théorique dans le processus de l'intervention psychosociale clinique par rapport à d'autres types d'interventions, comme celles conduites par des cabinets de conseil en gestion des ressources humaines, répond au souci de clarifier, $d^{\prime}$ abord pour nous-mêmes et ensuite, peut-être, pour d'autres, les relations $d^{\prime}$ implication mutuelle entre les principes éthiques, épistémologiques et méthodologiques qui orientent la démarche de recherche. En mettant en regard les modalités d'instauration du dispositif de l'intervention psychosociologique et les constantes de l'ordre social "racisé ${ }^{1}$ " qui prévaut dans les types de sociétés héritées de l'esclavage, nous espérons répondre aux interrogations sur la validité des connaissances produites par notre recherche. C'est en effet par l'analyse des conditions qui constituent l'objet de la demande d'intervention qu'il nous a été possible d'élaborer des connaissances de portée générale, complétant et enrichis- sant les travaux antérieurs sur la sociologie des rapports interethniques qui soulignent les contradictions et les tensions accompagnant nécessairement ces relations - expression de conflits entre valeurs idéales et pratiques sociales observables qui, " inspirées par les passions des hommes, s'éloignent fortement des idéaux proclamés (par les États démocratiques) " (Smeralda-Amon, 2002, p. 9). Les relations impliquées dans le processus de la recherche n'étant pas isolées des autres dimensions des ensembles dans lesquels elles s'inscrivent, l'énoncé qui donne sens à l'événement que représente l'intervention décrit également l'agencement des structures qui organisent I'action sociale sur un plan plus général.

Si I'on appréhende l'intervention comme une suite de moments se succédant dans le temps, la réinterprétation de la demande, initiant un travail d'analyse et de recherche théorique, ne correspond pas au premier moment de l'acte de consultation : " Dans ce premier moment, il est difficile, sinon impossible, de conduire une réflexion sur le sens de l'expérience et sur le rôle que l'on y a joué " (Dubost et Lévy, 2002, p. 414).

La réinterprétation de la demande ne peut donc se faire que dans l'après-coup, dans un deuxième temps. Cependant, les expériences d'intervention dans lesquelles nous avons été impliqués nous ont permis de voir que cela dépend de la posture choisie par l'intervenant dans la situation, des risques qu'il est prêt à prendre pour rester fidèle à son projet. Autrement dit, cette posture est référée à un ensemble de valeurs et de principes, qui constituent le support d'une identité professionnelle

1. Cette expression, qui désigne un ordre social référé à des critères raciaux, a été utilisée par Smeralda-Amon (2002). 
permettant au consultant de préserver son autonomie. "Par son autonomie, l'intervenant est constitué autre et ailleurs " (Ollivier, 2002). À partir de cet ancrage, des actes peuvent alors être posés, délimitant un espace de travail pour la production de nouvelles connaissances.

La manière dont nous sommes conduits à faire une place à un contenu $\mathrm{d}^{\prime}$ ordre axiologique pour établir des rapports de continuité et de discontinuité entre la pratique de l'intervention et la recherche scientifique correspond à une conception active de la connaissance (Boudon, 1986, p. 209) incompatible avec celle où la formulation de la demande laisse peu de place pour un travail $d$ 'analyse ; et où la pression exercée par les conditions interdit, au départ, la mise en place d'une démarche " collaborative ". C'est pourquoi nous considérons que le fait de ne pas présenter une méthode définie de façon unilatérale, mais de se donner le temps de la réflexion en posant, par exemple, comme préalable de réaliser un diagnostic de la situation, recourant à des techniques d'enquête sur le terrain, représente une option stratégique en rupture certes avec les principes habituels d'une démarche scientifique, mais cependant nécessaire. Dans ce sens, nous adhérons pleinement aux propos de Jean Dubost, qui critique cette tendance qui tendrait à faire croire que la démarche de l'intervention psychosociologique pourrait faire l'objet d'un processus de standardisation sur le plan méthodologique et technique (Dubost, 1987a, p. 12).

II ne s'agit pas, en effet, de mettre en place une démarche de recherche selon un plan conçu à l'avance. II s'agit plutôt pour le praticien-chercheur de réaliser un " saut dans l'inconnu ", d'être confronté à l'angoisse de ne rien trouver qui puisse lui permettre de saisir l'objet de la demande (Que veut-on faire ? Que veut-on changer ? Et pourquoi ?), et les facteurs générateurs du problème pour lequel son intervention est sollicitée (Dubost, 1987b). Face à la nouveauté de la situation, les savoirs issus des expériences antérieures de l'intervenant, tout comme la méthodologie et les techniques auxquelles il peut avoir recours ne présentent qu'un intérêt très limité. Ainsi, les actes qu'il pose sont davantage à considérer comme un " coup de force ", dans le sens où il s'agit souvent, dans ce premier moment, de ne pas déférer à l'injonction dans la commande adressée à I'intervenant 2 , tout en postulant l'existence $d$ 'autres possibilités contenues dans la situation et la volonté de les explorer, faisant ainsi une place à la théorie. En choisissant de suivre les "lois propres de sa posture » (B. Ollivier, 2002, p. 58), la présence du consultant crée donc une rupture et représente un événement qui ne se situe pas dans le cours habituel des choses. "Suscitant des interrogations, des craintes et des peurs, provoquant des prises de position, l'élaboration de nouveaux scénarios, donc en mobilisant des énergies, des sentiments et des affects, la présence du consultant initie un processus dans lequel intervenant et acteurs collaborent, dans des rôles distincts, à l'élaboration du sens " (A. Lévy, 1997, p. 17).

Cette façon de concevoir les liens existant entre les différents moments du processus de l'intervention trouve $\mathrm{d}^{\prime}$ autres appuis dans les travaux de 
Florence Giust-Desprairies. Dans son livre, Le désir de penser, Florence GiustDesprairies insiste sur l'importance que la démarche clinique attache à I'autonomie des personnes impliquées dans le processus de la recherche. II semblerait que I'indépendance du chercheur et sa liberté $d$ 'investigation, donc ses efforts pour se soustraire aux jeux de pouvoir et aux intérêts particuliers du commanditaire, soient une condition première garantissant la fiabilité des résultats de la recherche (Giust-Desprairies, 2004, p. 52).

La fonction que nous attribuons à des principes et à des valeurs dans le processus de l'intervention est à mettre en rapport avec la nécessité pour le consultant de prendre en compte dans son action la signification implicite de la demande aux différents registres sur lesquels elle s'exprime. Dans un travail commun, André Lévy et Jean Dubost (2002, p. 412) s'interrogent sur l'attitude à observer lorsque les connaissances produites par l'intervention vont à l'encontre de la demande d'un commanditaire animé par le désir d'accroître son pouvoir. En pareilles circonstances, ils estiment que, en tout état de cause, l'intervenant doit s'interdire toute tentative $d^{\prime}$ instrumentalisation des personnes et des collectifs auprès desquels il est sollicité. Mais, ce qui détermine cette posture, dans les termes où elle pourrait être énoncée au départ en résonance avec la position occupée dans la situation, peut être interprété comme une menace ou une remise en cause personnelle pour certains, $d$ 'où la nécessité pour le consultant de faire preuve d'une certaine retenue dans la relation, sans toutefois perdre de vue que c'est ce mode particulier de relation (non instrumentale, de coopération, d'aide...) que I'intervention cherche à établir qui permet de produire des changements significatifs relatifs à I'organisation du travail, aux rapports de pouvoir, au traitement des conflits, à la formation...

La visée de l'intervention psychosociologique est donc de participer à la construction d'une certaine forme de démocratie. Mais, comme nous espérons le montrer, ces finalités ultimes n'entretiennent pas de relations d'implication étroites avec la validité des connaissances produites. Car, " c'est dans la mesure où elles résultent $d^{\prime} u n$ processus relationnel et d'un apprentissage collectif conduisant à des évolutions psychologiques significatives, à la fois sur le plan intellectuel et affectif " (Dubost et Lévy, 2002, p. 392) que son projet peut prendre une signification politique dans la situation. Mais encore faut-il que ceux qui sont impliqués dans I'intervention soient prêts à s'engager dans cette voie.

Force est de constater que la perspective dans laquelle se place l'intervention psychosociologique est de considérer I'Autre dans son altérité propre. « Paradoxalement, cette compréhension du sujet comme un tout ne se réalise que dans une expérience singulière, dans un moment historique donné, où le sujet est saisi dans sa singularité, à travers les détails qui le rendent unique, voire aberrant, différent de tout autre (le Mélanésien de telle ou telle île) " (Lévy, 1997, p. 15). C'est ainsi que les connaissances produites dans la pratique de l'intervention psychosociologique sont moins déterminées dans leur fondement que celles issues des sciences humaines et sociales lorsqu'elles étudient les sociétés extra-occidentales, du fait que les conceptualisations qui ont dominé, et dominent encore la pensée sociale, sont I'héritage direct, comme le rappelle Juliette Smeralda-Amon, citant Copans, 
de « la perspective globalement qualifiée d'évolutionniste (qui) avait catégorisé I'Autre comme primitif " (SmeraldaAmon, 2002, p. 12).

Dans ce sens, l'encadrement de l'intervention psychosociologique par une éthique, définie à l'intérieur du champ de la discipline dont elle se réclame, disjoint la pratique de la recherche en situation du militantisme ou d'une certaine forme d'ethnocentrisme. Les principes éthiques qui orientent l'intervention participent ainsi à créer de multiples décalages dans la situation et dans la personne même du consultant, nécessaires à la pratique de la recherche. Dans les dispositifs qu'elle met en place, les relations de pouvoir constituantes du politique et partie prenante de la structuration de la situation peuvent alors être soumises à l'analyse, au même titre que les autres éléments qui font l'objet de la demande d'intervention ; d'où la dimension d'engagement que contient nécessairement la pratique de l'intervention psycho-sociologique.

Les deux interventions qui font I'objet de ce travail de réflexion montrent que lorsque les conditions spécifiques ne sont pas réunies pour la mise en place d'une démarche psychosociale clinique dans l'intervention, les connaissances produites par la recherche n'ont pas la même signification aux différents niveaux imbriqués de l'action sociale à laquelle elle participe. C'est ainsi que des connaissances nouvelles peuvent être produites, mais n'avoir aucun impact sur les conditions à partir desquelles elles ont été élaborées, comme ce fut le cas dans I'intervention dans la société $A$. Alors que I'intervention dans la société $B$ permet de voir que le processus initié a engagé les acteurs impliqués dans un travail de réflexion et $d^{\prime}$ abstraction se traduisant par une remise en question des savoirs et des convictions sur lesquels ils fondaient jusqu'à présent leur action. Dans ce sens, I'analyse comparative des résultats des deux interventions que nous présentons nous permet d'établir un lien entre le caractère $d$ ' « exemplarité " de l'intervention psychosociale clinique et la possibilité pour le consultant de subordonner son intervention à un objectif de recherche théorique avec l'assentiment plus ou moins explicite du commanditaire, car c'est de là que peut émerger et être prise en charge une demande de changement.

\section{EfFEtS DE LA PROBLÉMATIOUE RACIALE SUR LE DISPOSITIF D'INTERVENTION}

Les deux interventions auxquelles nous nous référons ont pour cadre des entreprises martiniquaises, dont l'une, se caractérisant par son implantation sur plusieurs sites, nous a conduits à nous déplacer en Guadeloupe, en Guyane et en Martinique. Ces entreprises, désignées par les lettres $A$ et $B$, ont en commun $d^{\prime}$ intervenir dans un secteur d'activité vital pour l'économie de ce département insulaire, celui des transports maritimes et aériens. Ce positionnement fait donc de ces organisations des "entreprises d'exception ". Nous utilisons ce terme pour souligner le fait que leurs activités conditionnent celles des autres secteurs économiques de leur environnement et se distinguent, dans leur fonctionnement interne, des autres organisations qui dépendent d'elles.

Dans l'une de ces interventions (I'entreprise A), nous avons été confrontés à une situation où le groupe qui formulait la demande d'intervention ne se considérait pas directement concerné par le processus qui devait être mis en place. N'ayant pas toujours eu les moyens de négocier les conditions de nos interven- 
tions, il nous est arrivé de participer à la conception d'actions de formation à partir des seules informations recueillies auprès des responsables ou de membres de l'encadrement. Les résultats obtenus dans ces conditions, comparés à ceux où nous disposions d'une plus grande « marge de liberté " (I'entreprise B) dans la structuration du dispositif, nous ont conduits à faire l'hypothèse que la possibilité pour la formation d'avoir des effets sur les structures ne dépend pas tant des orientations pédagogiques et des théories de l'apprentissage auxquelles le consultant réfère sa pratique que du pouvoir dont il dispose ou qu'on lui prête. Car, même s'il pouvait apparaître à l'exposé des problèmes posés que former des personnes à des " nouveaux métiers " implique de pouvoir se déplacer auprès d'elles pour connaître ce qu'il en est de leurs savoirs, de leurs désirs et de leurs souhaits d'évolution, il nous a aussi fallu reconnaître que la situation n'offrait pas toujours la possibilité d'élaborer une réponse en ces termes.

En prenant progressivement conscience de la façon dont la rigidité des structures faisait obstacle à l'affirmation de l'identité sociale du consultant et de son projet, nous avons fait I'hypothèse que I'analogie entre la structuration du dispositif et le contexte de l'intervention pouvait constituer une clef méthodologique pour l'analyse de la dynamique sociale instituant le cadre de l'intervention. En plaçant la problématique de l'accès à l'identité et de la reconnaissance sociale au centre du dispositif comme enjeu des dynamiques sociales, des échanges et des pratiques liés à la coopération entre acteurs, nous avons été amenés à reconstituer les conditions de l'apparition et de la réapparition du conflit, du malaise ou de la crise à l'origine de la demande.
Pour cela, nous avons dû intégrer dans I'analyse des dimensions souvent oblitérées dans la pratique, notamment dans celle des consultants qui puisent leurs référents du côté de "l'école du management ". L'une de ces dimensions est la hiérarchie sociale raciale. Dans les entreprises dirigées par des Blancs créoles, il est en effet rare de trouver des personnes de peau noire parmi le personnel de l'encadrement. C'est ainsi que nous avons été conduits à reconnaître le postulat concernant l'existence « d'un problème racial dans les anciennes colonies françaises en général » (Smeralda-Amon 2002), en observant comment ce problème, " intimement lié au préjugé de couleur ", se répercutait dans le cadre de l'intervention. Cette problématique étant "tabouisée ", peu d'études lui ont été consacrées jusqu'à présent, et il nous a fallu construire un cadre théorique pour donner sens aux effets que nous avons constatés dans la dynamique des échanges et des rapports entre les personnes impliquées dans le processus de l'intervention.

En effet, le fait de reconnaître que des individus de peau noire appartiennent à la communauté française n'empêche nullement les relations entre groupes en Martinique d'être marquées par des préjugés racistes plutôt qu'ethniques. C'est ainsi qu'Édith Kovats-Beaudoux (2002), pour ne pas avoir pris toute la mesure de ces effets dans l'étude réalisée sur les Blancs créoles de ce pays, est amenée, par manque de familiarité avec les autres groupes sociaux, à appréhender le réel qui leur correspond sous la forme d'un ensemble de formulations généralisantes où il est dit que : « La majorité de la population de couleur [...] est aliénée au sens complet du terme, en particulier vis-à-vis du Créole ", et que cela se traduit dans « des attitudes économiques, sociales et psychologiques " conditionnées 
par la « survivance des mentalités de l'esclavage ». En tant qu'ethnologue, accueillie dans une famille békée, pratiquant I'observation participante, sa participation à tous les grands événements qui ponctuent la vie du groupe, tels que mariages, baptêmes, enterrements, réceptions, repas de famille, etc., lui a permis de décrire avec finesse I'organisation de cette communauté. Mais en raison des défenses érigées par le groupe contre l'intrusion des gens de couleur sur son territoire, elle nous apprend comment elle fut " en (sa) qualité de blanche voulant s'intégrer au milieu créole » obligée de limiter ses contacts avec les autres groupes pour préserver son terrain d'étude ; et ceci sans toujours percevoir toutes les conséquences qu'une telle attitude induisait sur l'objet de la recherche.

Cette description de la hiérarchisation sociale nous a été très utile dans notre analyse des structures de pouvoir et de l'autorité à la base des rapports qui se nouent dans les cadres de travail. Le modèle type de l'organisation sociale de I'entreprise privée prend l'allure d'une structure hiérarchique plaçant en position haute les personnes blanches de peau, et en position basse, les personnes noires de peau (représentant la majorité de la population). Cette analyse théorique constitue la trame de pensée qui nous a permis $d$ 'analyser la demande $d$ 'intervention dans la société $A$, que l'on peut interpréter comme une demande de non-changement, c'est-à-dire visant à maintenir en l'état le fonctionnement des structures.

IMBRICATION DES STRUCTURES SOCIALES, DES STRUCTURES ORGANISATIONNELLES ET DES MODALITÉS D'INTERVENTION

L'intervention dans l'entreprise A illustre la manière dont la rigidité des structures sociales, les clivages entre les groupes en présence contribuent à faire obstacle aux actes qui s'imposent au consultant, à mesure que l'analyse de la demande dévoile les enjeux cachés, les dimensions multiples de la situation à partir de laquelle elle a émergé.

La commande consistait à réaliser un audit dans l'objectif de déceler les besoins en formation de personnels qui seraient prochainement embauchés. Cette étude devait intégrer un projet plus vaste, visant à créer une entité constituée par le regroupement de quatre entreprises indépendantes, mais œuvrant dans le même secteur d'activités. Pour réaliser cet audit, le commanditaire avait fait appel à un cabinet de conseil en ressources humaines dirigé par un consultant métropolitain. Sur le terrain, nous faisions équipe avec une consultante métropolitaine, elle-même responsable du projet.

Nous avons appris au cours d'entretiens avec les responsables de ces entreprises, représentant les personnes ressource que l'on nous avait indiquées, qu'elles avaient, jusqu'à présent, recours aux services de ces personnels dans le cadre de ce qui s'apparentait à un contrat de prestation de services. Ces entretiens ont permis de recueillir les informations dont nous avions alors besoin pour élaborer le plan de formation, visant à préparer cette main-d'œuvre aux nouveaux métiers qui allaient être créés dans le cadre de la prochaine mise en service d'une nouvelle plate-forme logistique.

Nous avons rencontré tous les dirigeants des entreprises associées à ce projet. Parmi eux, ne figurait qu'un seul Martiniquais de phénotype noir, gérant une société de taille très modeste, n'employant qu'un seul salarié. Les autres dirigeants étaient des Békés (Blancs créoles) ou des métropolitains et ils étaient à la tête d'entreprises nettement plus 
grandes, employant plusieurs salariés dont des cadres, spécialistes du transport et de la logistique. La plupart de ces personnels avaient déjà travaillé auparavant sur $d$ 'autres plates-formes logistiques avant leur arrivée dans ces entreprises. Ils connaissaient bien I'environnement de ces nouveaux métiers.

Notre participation au travail d'étude s'est arrêtée au stade du recueil des données nécessaires à l'élaboration du plan de formation des futurs salariés de la nouvelle entreprise. Nous n'avons donc pas eu l'occasion de rencontrer les salariés noirs pour qui nous étions censés élaborer ce plan de formation. Les responsables de ces entreprises n'avaient pas jugé utile de les faire participer à ce travail. De ce point de vue, ils considéraient les connaître suffisamment pour savoir ce qui était bon pour eux. Un des dirigeants d'origine métropolitaine participant à ce travail de réflexion a tenté d'exprimer un point de vue contraire, indiquant qu'il n'était pas hostile à l'idée d'intégrer des Noirs à des postes d'encadrement, mais sa position ne fut prise en compte ni par les autres dirigeants békés ni par la consultante blanche qui faisait équipe avec nous.

La suite de l'intervention, qui s'est donc poursuivie sans notre participation, en raison de tensions dans l'équipe des consultants, a montré que si l'intervention avait permis aux dirigeants de prendre des décisions en vue de former les personnes nouvellement embauchées, elle n'avait pas pour autant contribué à les aider à résoudre les difficultés qu'ils appréhendaient au regard de l'intégration de ce personnel. Des mouvements de grèves qui ont émaillé la vie de l'organisation à l'issue de la période de l'intervention nous ont conduits à poursuivre ce travail d'investigation par d'autres moyens. C'est ainsi que la confrontation des données du terrain avec d'autres éléments de réflexion nous a permis de faire l'analogie entre les structures organisationnelles et celles de la société de plantation (Galap, 1997, p. 155-171). II $\mathrm{s}^{\prime}$ agissait pour nous de traduire, à travers la prise de conscience des effets du processus organisationnel sur le processus de l'intervention, l'idée d'une " racisation " des structures de l'entreprise. Car le fait pour l'équipe des consultants $\mathrm{d}^{\prime}$ accepter de s'interdire de rencontrer les salariés noirs afin de recueillir leurs impressions sur les modalités de mise en œuvre des formations qui leur étaient destinées réduisait les limites de l'intervention. Celle-ci était ainsi ramenée au seul objectif de la rédaction d'un cahier des charges, c'est-à-dire à un travail d'ingénierie pédagogique. Ainsi structuré, le dispositif d'intervention n'a pas permis que l'on puisse s'interroger sur les implications possibles de l'intégration de ces personnes dans les structures sociales de I'organisation. Or, ainsi que nous avons cherché à le montrer, l'analyse permettait d'interpréter l'exclusion de ces personnes par la volonté d'un groupe de dirigeants de refuser de les reconnaître comme des participants à part entière du processus organisationnel. Nous estimons donc qu'un travail centré sur l'analyse des conditions d'intégration de ces salariés, mené avec l'ensemble de ces personnes, aurait permis d'anticiper certains de ces effets, contre lesquels ces personnes n'ont pas manqué de réagir, sans toujours avoir conscience de ce qui était (et est toujours) à l'origine de leur mal-être.

On peut en effet noter que les entreprises dirigées par les Békés font appel exclusivement à des cabinets métropolitains ou békés. Dans le cas ici cité, ce qui nous a permis d'intervenir est le fait 
d'être engagé dans une équipe composée de métropolitains. Les consultants martiniquais sont très conscients de cette réalité, et ils en tiennent compte pour leur positionnement dans leurs interventions. Ils savent qu'ils ne peuvent accéder au marché de la formation que sous couvert de la relation qui les lie à des prestataires de services métropolitains ou békés. C'est ainsi que faute de subordonner leurs interventions à un travail d'ordre théorique, ils contribuent à la reproduction des inégalités que sécrète l'ordre culturel (politique, économique et social) qui constitue les fondements des structures organisationnelles.

Notre intervention dans la société B a pris une tout autre tournure. Elle dure depuis bientôt trois ans, et se poursuit au moment où nous rédigeons cet article. Elle a permis de mettre en évidence que des structures sociales moins rigidement structurées, "caractérisées par une multiplicité des repères symboliques, parfois contradictoires, se traduisant par de la souffrance et de l'angoisse engendrées par cette dilution des références " (Lévy, 1997), constituent des conditions susceptibles de favoriser l'émergence d'une demande d'aide.

Sans pouvoir présager de ses effets, nous ne pouvons présenter ici que de façon très sommaire la démarche d'intervention, ses temps forts et notre analyse des rapports entre le processus de formation et les événements ayant eu lieu dans l'organisation : intégration du personnel, changement des procédures de recrutement, " accord de méthode " décidé en commun par le syndicat et la direction pour le règlement des conflits, grève, exclusion du responsable d'unité par les représentants du personnel.

Lorsque nous avons rencontré le directeur de l'entreprise, originaire de la métropole, celui-ci souhaitait mettre en place une formation en management pour les responsables des trois établissements qu'il dirige en Martinique, Guadeloupe et Guyane. Cela faisait deux ans qu'il avait été nommé à ce poste par l'un des actionnaires, assurant durant cette période la gérance de ce groupement d'entreprises. L'actionnaire qu'il représentait devait bientôt céder sa place à un autre actionnaire du groupement. Son passage à ce poste lui avait permis de constater que certains responsables des établissements implantés dans chacun des départements ne s'impliquaient pas dans la gestion des affaires sociales de l'unité qu'ils dirigeaient, laissant ce rôle au directeur. II voulait y remédier, d'abord en les formant aux techniques du management et ensuite en leur attribuant un statut de cadre de façon à officialiser leur fonction. Cette démarche représentait, de son point de vue, un moyen pour amener ces responsables d'établissement à jouer un rôle plus actif auprès de la direction.

Dans la discussion qui s'est ensuite engagée sur l'opportunité de la formation, nous avons fait part de notre scepticisme sur la pertinence de la démarche pour produire les changements souhaités. Car il apparaissait déjà qu'il ne s'agissait pas seulement de créer une équipe de direction autour du directeur pour le soutenir dans son action, mais aussi et surtout de prévenir le déclenchement d'une grève.

Dans un premier temps, nous avons proposé au directeur de réaliser un audit de l'organisation, préalablement à la mise en place de toute formation ou de toute action visant à rétablir la situation. Ceci afin de mieux comprendre les difficultés rencontrées par les personnes impliquées. Le directeur de l'entreprise accepta notre proposition. Peu de temps avant le 
démarrage de l'intervention, il nous apprendra que, pour la première fois dans I'histoire de l'entreprise, la personne qui bientôt devait le remplacer dans ses fonctions était d'origine martiniquaise. S'il estimait que la nomination d'un Martiniquais à ce poste représentait un tournant important dans I'histoire de l'entreprise, il n'était pas sûr, se fondant pour cela sur son expérience, que le personnel partage la même vision que lui sur la dimension de cet événement.

Pour répondre aux objectifs définis par le directeur, nous avons choisi d'associer à ce travail un consultant en management. Cette décision visait pour beaucoup à apaiser les craintes de notre commanditaire de s'engager dans une aventure dont il mesurait mal les retombées éventuelles.

La présentation d'un support d'enquête aux dirigeants pour approbation répondait à leur volonté de prendre toutes les précautions dont ils pourraient s'entourer afin d'éviter un rejet de l'intervention par le personnel. Sous couvert de la transparence, cette attitude suffisait déjà à montrer le pouvoir qu'ils attribuaient au personnel. Le démarrage de l'intervention, prévue pour être reproduite dans les autres établissements de l'entreprise, ne nous permettait pas d'être très optimistes quant à sa durée dans le temps, compte tenu de la prudence qu'affichaient alors les dirigeants, qui pouvait laisser craindre qu'ils seraient prêts à abandonner leur projet de changement s'il rencontrait la moindre résistance venant du personnel.

Nous avons associé tous les niveaux de la hiérarchie, dont les dirigeants, à ce travail. Dans ces entretiens, il est apparu que le personnel administratif, comme les dirigeants, considérait les personnels de la production comme des personnes peu " instruites " et " assez frustes ", avec lesquelles il était difficile d'établir un dialogue. De leur côté, le groupe des salariés de la production, installé dans un établissement séparé de l'administration et composé uniquement d'hommes, disait ne pas avoir de contact avec les gens de l'administration; ils se représentaient eux-mêmes comme les seules personnes dont l'activité était essentielle au fonctionnement de l'entreprise.

Les salariés de I'unité de production que nous avons vus en entretien disaient ne pas comprendre le sens de notre présence dans l'entreprise, puisqu'ils ne rencontraient aucune difficulté dans leurs rapports. Ils présentaient l'unité dans laquelle ils travaillaient comme une grande "famille " où tout le monde se connaissait. Un grand nombre de ces salariés avaient, en effet, dans leurs fonctions, succédé à leur père ou encore avaient un frère dans l'entreprise. D'autres, au contraire, dénonçaient, à mots couverts, les " arrangements " entre le syndicat et la direction, et cette " loi du silence " qui leur était imposée. Mais tous présentaient leur travail comme un métier facile, pour l'exercice duquel il n'était pas nécessaire d'avoir un diplôme. Être titulaire du permis poids lourd constituait le seul critère exigé pour être embauché dans l'entreprise. Aussi, ils ne voyaient pas l'utilité de participer à des formations.

Si notre présence dans l'entreprise était voulue par les dirigeants, elle ne semblait être que tolérée par certains, notamment pour le représentant syndical qui a tenu à nous signifier clairement qu'il $\mathrm{s}^{\prime}$ agissait pour les salariés de faire preuve de courtoisie à l'égard de leur direction. Nous avons vite compris qu'il s'agissait $\mathrm{d}^{\prime}$ adresser une menace à peine voilée à la direction de l'entreprise. II voulait nous faire comprendre que le fait que le nouveau 
directeur soit martiniquais ne changerait rien aux rapports qu'ils entretenaient avec leur direction, comme d'ailleurs avec le responsable de l'unité de production. Pour lui, cette entreprise leur appartenait et il ne fallait pas que la direction puisse penser qu'elle avait tous les droits.

Après analyse et restitution des résultats de l'enquête au commanditaire et au personnel, nous avons proposé un plan d'intervention au directeur de l'entreprise qui visait à intégrer, dans la commande, ces demandes qui remontaient du terrain. Pour cela, nous avons dû nous opposer au directeur et prendre le risque de perdre un "marché ". Car nous avons très vite compris que nous ne pouvions pas nous laisser enfermer dans le rôle de "prestataire de services " que le directeur de l'entreprise voulait nous donner, si l'on voulait aider ces personnes à dépasser leurs difficultés.

Notre analyse des discours de représentations laissait en effet apparaitre que cette représentation de l'unité de production comme une "grande famille " était loin de traduire une image d'harmonie. La " loi du silence " et les " petits arrangements " qui l'accompagnaient nous faisaient davantage penser à l'idée que nous avons des structures mafieuses, du type de celles de la Cosa nostra. Le responsable syndical, ayant lui-même succédé à son père dans ses fonctions et réussi à faire intégrer son frère dans l'entreprise, transgressant ainsi la règle écrite qui voulait qu'il n'y ait pas plus d'une personne d'une même famille dans l'entreprise, s'enorgueillissait que le personnel ait pu faire inscrire dans le protocole d'accord de l'entreprise qu'au départ d'un salarié à la retraite, son fils puisse lui succéder à son poste. Cette règle fonctionnait sur le principe d'une « règle fondamentale " subordonnant toutes les autres, donnant au syndicat un pouvoir illimité censé lui permettre de régler toutes les difficultés de fonctionnement (recrutements de nouvelles personnes, promotions, rapports avec la hiérarchie...).

Dans les propos du responsable syndical, comme dans ceux des salariés placés au plus bas niveau de la hiérarchie sociale, il n'était nullement fait référence à la fonction des chefs d'équipe comme représentants de l'autorité au sein de l'organisation. Dans leurs représentations, deux figures d'autorité émergeaient : celle du syndicat et celle du responsable d'unité, encore qu'à ce dernier on n'attribuait qu'un pouvoir assez limité, car on savait que s'il venait à se montrer « trop arrogant ", on n'hésiterait pas à lui interdire de pénétrer dans l'établissement.

Personne n'ignorait que le pouvoir réel était détenu par le personnel et le syndicat chargé de le représenter, mais il n'était pas facile pour tout le monde de I'admettre, en particulier pour le directeur de l'entreprise. Pour ce dernier, comme pour son prédécesseur, il était plus commode de mettre en cause les compétences en " management " du responsable d'unité dans les dysfonctionnements organisationnels. Cette attitude leur permettait de ne pas reconnaître leur impuissance face à des phénomènes dont ils avaient des difficultés à comprendre les causes.

Dans ces conditions, nous n'avions d'autre choix que celui de déférer à la demande de la direction de mettre en place une formation en management à I'intention des responsables d'unité. Mais, conscients que cette démarche ne pouvait présenter qu'un intérêt limité compte tenu de la nature des difficultés que rencontrait l'organisation, nous avons insisté sur la nécessité d'insérer la formation des 
responsables d'unité dans un dispositif plus large. Nous estimions alors que :

- former uniquement le responsable d'établissement aux techniques de management n'aurait qu'un impact limité sur le processus organisationnel si on négligeait de relayer son action au niveau de la hiérarchie intermédiaire, c'est-à-dire en ne repositionnant pas les chefs d'équipe dans un rôle d'encadrants et d'animateurs d'équipe auprès des personnels sous leur autorité ;

- établir les procédures de recrutement et de promotion sur des critères et modalités moins spécifiques à l'organisation (niveau de formation, entretien de sélection, tests d'évaluation...) permettrait de réduire les effets négatifs de la variable " familiale " et l'influence des phénomènes de groupe sur le fonctionnement des instances dirigeantes de l'organisation (chefs d'équipe, responsables d'établissement, direction) ; - initier une action visant à enrichir l'éventail des activités des salariés, sur la base des souhaits et des centres d'intérêt des individus, pourrait engager un processus de développement des compétences liées à l'espérance d'une mobilité sociale susceptible de profiter à l'organisation ou de se concrétiser dans un projet de reconversion.

$C^{\prime}$ est ainsi que nous avons pu établir deux groupes de formation. Le premier groupe était composé par les responsables d'unité des différents établissements de la Martinique, de la Guadeloupe et de la Guyane, alors que le second comprenait uniquement des salariés de l'établissement de la Martinique. Hormis pour la formation des responsables d'unité, les sessions de formation se tenaient hors des murs de l'entreprise.

Bénéficiant désormais du soutien $d^{\prime} u n e$ équipe composée d'un consultant dans le domaine du management et de formateurs, notre place dans le dispositif de la formation évolua sensiblement au cours des trois années qui ont suivi le démarrage de l'intervention. D'un rôle d'enquêteur, au début de l'intervention, et d'intervenant dans la formation des responsables d'unité, nous avons évolué vers un rôle de coordinateur de projet et de conseil auprès de la direction. Moins concernée par les dimensions pédagogiques de l'intervention, cette position d'écoute et de conseil nous a alors permis de mener un travail d'analyse et de réflexion avec les personnes auprès desquelles nous intervenions. C'est ainsi que dans le groupe composé des chefs d'équipe et de salariés de la base, et auquel participaient également les représentants du personnel, ce travail d'analyse et de réflexion en commun a permis de comprendre différemment les blocages constatés dans les rapports entre le responsable d'unité et son équipe. Ils paraissaient remonter à une histoire passée coïncidant avec l'arrivée du responsable d'unité dans l'entreprise. Ce dernier, ancien militaire de carrière, avait dès le départ voulu changer le fonctionnement dans I'entreprise en imposant une discipline très stricte contre laquelle le personnel s'était aussitôt mobilisé, faisant barrage à son autorité. Mais avec le recul, on pouvait reconnaître plus facilement les raisons qui justifiaient l'attitude de ce responsable et la part qu'il a prise dans l'initiation d'un processus de changement qui apparaissait désormais comme inéluctable. Ces considérations sur les concessions que le responsable d'unité avait dû faire sous la pression du personnel et du syndicat constituaient une explication de son positionnement actuel et du climat social au sein de l'entreprise. Ces propos représentaient également une critique plus ou moins explicite de la situation. 
Aussi, lorsque nous avons été consultés par la direction, pressée de supprimer les obstacles éventuels qui pourraient se dresser contre son projet de changement, sur la décision de promouvoir le responsable syndical de l'entreprise dans un poste d'encadrement, nous lui avons fait part de la volonté des salariés de voir établir une égalité de traitement entre les personnes pour plus de transparence dans les rapports. Sur nos conseils, les consultations menées auprès de certains membres du groupe, notamment des chefs d'équipe, ont conduit alors la direction à prendre la décision de nommer quelqu'un d'autre que le responsable syndical dans le poste de chef d'équipe nouvellement créé. Et, contrairement aux appréhensions de la direction, cette décision ne déclencha aucun conflit au sein de l'entreprise, le positionnement des chefs d'équipe contribuant au contraire à réduire les liens de dépendance informelle entre le syndicat et la direction.

Le déroulement de notre intervention dans I'entreprise B montre comment notre propre prise de conscience concernant les enjeux de la situation a facilité un processus semblable chez les membres de l'entreprise. Pour parvenir à ces résultats, il nous a fallu adopter une attitude plus critique envers les exigences de notre commanditaire qui souhaitait utiliser notre présence pour faire passer des « messages " auprès du groupe constitué par les responsables d'unité, pour lesquels on souhaitait mettre en place une formation " en management ". L'opportunité qui nous a été donnée de pouvoir adopter une position d'écoute et d'analyse, loin de nous désengager du dispositif, nous a au contraire conduits à assumer nos responsabilités dans la production et la diffusion de connaissances nouvelles sur l'organisation, tout en mettant en évidence le rôle de la direction dans les difficultés de l'entreprise.

Par ailleurs, le fait pour nous d'intervenir dans la structuration méthodologique et technique du processus, en prenant des initiatives ou en faisant des propositions, a permis de faire en sorte que le cadre constitué par les structures organisationnelles apparaisse comme un lieu où il était désormais possible de faire coïncider des paroles avec des actes. Car dans la mesure où les acteurs avaient le sentiment que les engagements pris seraient tenus, ils pouvaient entrevoir la possibilité de mettre en place, avec quelque chance de succès, des stratégies de mobilité professionnelle. Chose qui restait exclue dans la société $A$ où, faute de pouvoir prendre en compte l'influence des facteurs déterminant les comportements, on pouvait toujours continuer à considérer que les revendications salariales du personnel, pourtant très bien rémunéré, constituaient une attitude irrationnelle.

\section{Bibliographie}

Boudon, R. 1986. L'idéologie, Paris, Fayard.

DuBost, J. 1987a. " Sur les sources techniques de l'intervention psychosociologique et quelques questions actuelles ", Connexions, n ${ }^{\circ} 49$, Paris, Epi.

DuBost, J. 1987b. L'intervention psychosociologique, Paris, PUF.

DuBost, J. ; LÉVY, A. 2002. "Rechercheaction et intervention ", Vocabulaire de psychosociologie, Toulouse, érès.

GalaP, J. 1997. " En-quête d'identité : culture, migration, l'exemple antillais ", 2001, L'odyssée du 
placement familial ou l'illusion $d u$ retour, Toulouse, érès, Grape.

GIUSt-DespraIRIES, F. 2003. L'imaginaire collectif, Toulouse, érès.

Giust-Desprairies, F. 2004. Le désir de penser, Paris, Téraèdre.

Kovats-Beaudoux, E. 2002. Les Blancs créoles de la Martinique, Paris, L'Harmattan.

LÉVY, A. 1997. Sciences cliniques et organisations sociales, Paris, PUF.

OllivieR, B. 2002. "Autonomie ", Vocabulaire de psychosociologie, Toulouse, érès.

SmeRALDA-AmON, J. 2002. La racisation des relations intergroupes ou la problématique de la couleur, le cas de la Martinique, Paris, L'Harmattan.

\section{RÉSUMÉ}

Cet article cherche à montrer comment toute action de changement dans des entreprises martiniquaises et guadeloupéennes se heurte aux résistances provoquées par les structures et les clivages de nature raciale profondément ancrés dans la culture antillaise, hérités du colonialisme et de l'esclavage, qui conditionnent dans l'entreprise les identités, les rapports hiérarchiques, les modes de division du travail, et les conduites individuelles et collectives. Dans cette situation difficile, le consultant est mis en question dans son identité personnelle et professionnelle. Afin de pouvoir maintenir néan- moins la position de tiers requise par la démarche d'intervention psychosociologique, il doit s'appuyer sur des valeurs et des convictions d'ordre éthique, mais aussi sur de solides bases théoriques (théorie de l'intervention, analyses de la société martiniquaise et de son histoire).

\section{MoTS-CLÉS}

Intervention psychosociologique, culture antillaise, relations interethniques.

\section{SUMmARY}

This article tries to show how any action of change in Martinique and Guadelupian enterprises collides in resistances provoked by the racial structures and cleavages profoundly anchored in the culture, inherited from colonialism and slavery. They condition within the enterprise the identities, the hierarchical relationships, the modes of division of labour and individual and collective behaviours. In this difficult situation, the consultant is questioned in his personal and professional identity. To be able to maintain nevertheless the position of third party, he should lean on values and convictions of ethical order, but also on robust theoretical bases (theory of the intervention, analyses of the Martiniquian society and its history).

\section{KeYWORDS}

Psychosociological intervention, antillaise culture, interethnic relationships. 